\title{
Mutation analysis of $\beta$-thalassemia in East-Western Indian population: a recent molecular approach
}

This article was published in the following Dove Press journal:

The Application of Clinical Genetics

II May 2017

Number of times this article has been viewed

\author{
Parth S Shah' \\ Nidhi D Shah² \\ Hari Shankar P Ray ${ }^{3}$ \\ Nikunj B Khatri ${ }^{3}$ \\ Ketan K Vaghasia ${ }^{3}$ \\ Rutvik J Raval ${ }^{4}$ \\ Sandip C Shah ${ }^{3}$ \\ Mandava $\vee \mathrm{Rao}^{5}$ \\ 'Department of Medicine, Lahey \\ Hospital and Medical Center, Boston, \\ MA, ${ }^{2}$ Department of Pediatrics, \\ Nassau University Medical Centre, \\ New York, NY, USA; ${ }^{3}$ Supratech \\ Micropath Laboratory and Research \\ Institute, ${ }^{4}$ Department of Zoology, \\ ${ }^{5}$ School of Sciences, Gujarat \\ University, Ahmedabad, Gujarat, India
}

Correspondence: Sandip C Shah Supratech Micropath Laboratory and Research Institute, Kedar Complex, Parimal Garden, Ahmedabad 380006, Gujarat, India

Tel +9l 9824030580

Email supratech18@gmail.com
Background: $\beta$-Thalassemia is the most prevalent genetic disorder in India. Its traits and coinheritance vary from mild to severe conditions, resulting in thalassemia minor, intermediate, and major, depending upon many factors.

Purpose: The objective of this study was to identify the incidence of $\beta$-thalassemia traits, their coinheritance, and mutations, as well as to support the patients already diagnosed with $\beta$-thalassemia in East-Western Indian population for better management.

Patients and methods: Seventy-five referral cases for $\beta$-thalassemia were analyzed for various $\beta$-thalassemia traits, heterozygosity, and homozygosity conditions. Blood phenotypic parameters using cell counter and capillary electrophoresis were investigated. Analyses of eight common mutations of thalassemia in India were carried out using polymerase chain reaction-amplification refractory mutation system, end point polymerase chain reaction, and DNA sequencing methods. Results: Of these (75) referral cases from East-Western Indian region, 68 were positive for $\beta$-thalassemia (90.67\%). The majority of case types were of $\beta$-thalassemia minor $(49,65.33 \%)$, followed by $\mathrm{HbE}$ traits $(6,8.0 \%)$ and $\beta$-thalassemia major, including heterozygous and homozygous $(5,6.66 \% ; 4,5.33 \%)$ types and then $\mathrm{HbE}$ homozygous $(2,2.66 \%)$, as well as one each of the $\mathrm{HbE} / \beta$-thalassemia and $\mathrm{HbD} / \beta$-thalassemia $(1,1.34 \%)$ combination. Mutation analysis also revealed that the highest frequency of mutation was c. $92+5 \mathrm{G}>\mathrm{C}(41,60.29 \%)$ followed by deletion $619 \mathrm{bp}(9,13.23 \%)$ and c.79G $>$ A $(8,11.76 \%)$ in our study group. Five cases (nos. 24, 27, 33, 58, and 71) exhibited coinheritance between $\beta^{0} / \beta^{+}(2), \beta^{0} / \beta$ D (1), and c.124_127delTTCT $/ \beta^{+}$or $\beta^{0}$ (2) affecting the Rajasthani and Gujarati populations in our study of the Western region of India. Conclusion: We strongly recommend these Western populations for genetic screening before adopting reproductive technologies and interracial marital relations.

Keywords: $\beta$-thalassemia traits, coinheritance, hematogram, capillary electrophoresis, PCRARMS, DNA sequencing, mutation analysis, East-Western India

\section{Introduction}

$\beta$-Thalassemia is one of the hemoglobinopathies belonging to a class of genetic disorders. It occurs due to mutation in $\beta$-gene of autosomal chromosome $11 .^{1}$ The incidence of $\beta$-thalassemia trait in India is $3.3 \%$ with $1 \%-7 \%$ of couples being affected annually. ${ }^{2}$ Approximately 300 mutations would occur in this type, affecting $\beta$-chain globin synthesis. If the synthesis of two $\beta$-chains is absent $\left(\beta^{0} / \beta^{0}\right)$, the person has $\beta$-thalassemia major (Cooley's anemia). This condition follows severe microcytic and hyochromic anemia. The person requires lifelong transfusion. $\beta$-Thalassemia minor is asymptomatic and results in microcytosis and mild anemia and $\mathrm{HbA} 2$ level increases, designated as $\beta^{+} / \beta$ or $\beta^{0} / \beta$. Usually thalassemia intermedia is a condition between the major and minor forms depending on the severity of the anemic condition $\left(\beta^{+} / \beta^{+} /\right.$or $\left.\beta^{0} / \beta^{+}\right)$among other cases..$^{3,4}$ 
Others are $\mathrm{HbE}$ trait, $\mathrm{HbE}$ homozygous, $\mathrm{HbD} / \beta$-thalassemia, and $\mathrm{HbE} / \beta$-thalassemia hemoglobinopathies. The latter one, $\mathrm{HbE} / \beta$-thalassemia, is maximum in Thailand. ${ }^{5}$ The gene mutation takes place in another one of $\beta$-gene only in addition to $\beta$-thalassemia minor/allele $\left(\beta^{0} / \beta\right.$ or $\left.\beta^{+} / \beta\right)$, leading to coinheritance. In India, such coexisting $\mathrm{HbE} / \beta$-thalassemia and $\mathrm{HbD} / \beta$-thalassemia are less debated and occur in some parts of India, Pakistan, and Iran. ${ }^{6,7}$ Recently, a report was published in Eastern Indian population about the status of thalassemia and hemoglobinopathies and suggested that more such studies are necessary in other regions of India. ${ }^{8}$ Prevalence of common hemoglobinopathies in Gujarati population was documented by Patel et $\mathrm{al}^{9}$ in screening programs, where $\beta$-thalassemia minor cases were maximum comparatively. Hence, we report $\beta$-thalassemia, $\mathrm{HbE}, \mathrm{HbD}$ traits, and their coinheritance as well as the mutation analysis of $\beta$-thalassemia distribution in East-Western Indian population using electrophoresis, polymerase chain reaction-amplification refractory mutation system (PCR-ARMS), end point PCR, and gene sequencing technology in our study.

\section{Patients and methods}

\section{Patient selection}

Blood samples of 75 referral cases of both sexes varying in age from 6 months to 38 years were collected from Gujarat (17), Rajasthan (40), Maharashtra (7), Assam (3), and West Bengal (6) in India for $\beta$-thalassemia testing after duly filling the patient consent form in our Supratech Micropath Research Institute, Ahmedabad. These patients were referred at random. This project was approved by Human Ethical Committee (HEC) of Gujarat University, Ahmedabad (GU/ HEC-001/15), in 2015 for investigation.

\section{Hematological analysis and DNA extraction}

Hematogram report was carried out on CELL DYN RUBY automated cell counter. Hemoglobin $(\mathrm{Hb})$ levels were estimated by Sebia Capillary 2 Flex piercing electrophoresis. The DNA was extracted from $2 \mathrm{~mL}$ of EDTA blood using PerkinElmer Prepito DNA Blood 250 Kit automatic machine. The kit was used according to the manufacturer's instructions. The extracted genomic DNA was used as a template and was kept at $4^{\circ} \mathrm{C}$ until further use after routine DNA check.

\section{Amplification, purification, and cycle sequencing}

The primers were synthesized from the Eurofins, India. The amplification reaction was performed in Veriti Thermal Cycler. The PCR products were loaded on a $2.5 \%$ agarose gel, and the amplicons were visualized under ultraviolet transillumination after staining with ethidium bromide. PCR product cleanup using USB ExoSAP-IT kit (Affymetrix, Santa Clara, CA, USA) and cycle sequencing using BigDye TerminatorV3.1 cycle sequencing kit (Applied Biosystems, Foster City, CA, USA) were used for further mutation identification.

\section{Mutation analysis}

The $\beta$-globin gene mutations were first characterized using two sets of allele-specific PCR-ARMS to detect eight common mutations in India including c.92+5G $>\mathrm{C}$, deletion 619 bp, c.79G>A (p.E27K), c.47G>A (p.Trp16Ter), c.364G $>$ C (p.E122Q), c.27_28insG, c.51delC, and c.124_127delTTCT. Unknown $\beta$-thalassemia genes were further characterized by direct DNA sequencing using 3500 Genetic Analyzer Applied Biosystems (ABI) for all coding regions and exon-intron boundaries to detect uncommon point mutations and small rearrangements in the $\beta$-globin gene. The c. $92+5 \mathrm{G}>\mathrm{C}$ mutation was detected by Sanger sequencing and PCR-ARMS, and deletion 619 bp was done by end point PCR (gel electrophoresis). Other mutations were analyzed only by Sanger sequencer. The data were analyzed using CodonCode Aligner v5.0.2 (CodonCode Corporation, Centerville, MA, USA) and Mutation Surveyor v5.0 (Softgenetics, State College, PA, USA). Mean and percentage were calculated wherever necessary.

\section{Results}

\section{$\beta$-Thalassemia and other traits}

Referral cases of 75 at our Supratech Micropath Research Institute, Ahmedabad, from 2015 to 2016 were analyzed for $\beta$-thalassemia and other traits based on $\mathrm{Hb}$ levels blood indices and mutation analysis from different parts of India. The affected (68) contributed $90.67 \%$ of the referral cases. High percentage $(65.33 \%)$ had $\beta$-thalassemia followed by $\mathrm{HbE}$ trait (8\%) and $\beta$-thalassemia major (heterozygous $6.66 \%$; homozygous 5.33\%). Others were HbE homozygous (2.66\%), $\mathrm{HbE} / \beta$-thalassemia, and $\mathrm{HbD} / \beta$-thalassemia contributed only $1.34 \%$ each. Thus, 49 cases $(65.33 \%)$ had $\beta$-thalassemia minor followed by $\mathrm{HbE}$ trait $(8 \%)$ and $\beta$-thalassemia major (compound heterozygous $6.66 \%$ and homozygous $5.33 \%$ ). Two were $\mathrm{HbE}$ homozygous (2.66\%) and $\mathrm{HbE} / \beta$-thalassemia and $\mathrm{HbD} / \beta$-thalassemia contributed only $1.34 \%$ each. These hemoglobinopathies are well supported by increased levels of mean HbA2 $(08.66 \%)$ with decreased mean corpuscular hemoglobin $(\mathrm{MCH})$, mean corpuscular volume values, and altered mean $\mathrm{HbD}$ (1.25\%), $\mathrm{HbF}$ (7.21\%), and $\mathrm{HbE}(2.73 \%)$ levels, measured by capillary electrophoresis (Tables 1 and 2 and Figure 1). 
Table I Classification of thalassemia traits in our study

\begin{tabular}{|c|c|c|c|c|}
\hline Nos & Types of thalassemia traits & Characteristics & Cases & Percentage \\
\hline $\mathrm{I}$ & $\beta$-thalassemia minor & Increased $\mathrm{HbA} 2$ with $\downarrow \mathrm{MCV}$ and $\uparrow \mathrm{RDW}$ & 49 & 65.33 \\
\hline 2 & HbE trait & $\uparrow \mathrm{HbA} 2 / \mathrm{E}, \mathrm{HbF}$, and $\downarrow \mathrm{HbA}$ and $\uparrow \mathrm{RDW}$ & 6 & 8.00 \\
\hline 3 & $\beta$-thalassemia major (compound heterozygous) & $\uparrow \mathrm{HbA} 2$ with $\downarrow \mathrm{MCV}, \uparrow \mathrm{HbF}$, and $\uparrow \mathrm{RDW}$ & 5 & 6.66 \\
\hline 4 & $\beta$-thalassemia major (homozygous) & $\uparrow \mathrm{HbA} 2$ with $\downarrow \mathrm{MCV}, \uparrow \mathrm{HbF}$, and $\uparrow \mathrm{RDW}$ & 4 & 5.33 \\
\hline 5 & HbE homozygous & $\uparrow \mathrm{HbA} 2 / \mathrm{E}, \mathrm{HbF}$, and $\downarrow \mathrm{HbA}$ and $\uparrow \mathrm{RDW}$ & 2 & 2.66 \\
\hline 6 & Combination of $\mathrm{HbE} / \beta$-thalassemia & $\uparrow \mathrm{HbA} 2 / \mathrm{E}, \mathrm{HbF}$, and $\downarrow \mathrm{HbA}$ and $\uparrow \mathrm{RDW}$ & I & 1.34 \\
\hline 7 & Combination of $\mathrm{Hb}-\mathrm{D} / \beta$-thalassemia & $\uparrow \mathrm{HbAD}, \mathrm{HbA} 2$, and $\mathrm{HbF} \uparrow$ (sometimes) and $\uparrow \mathrm{RDW}$ & I & 1.34 \\
\hline
\end{tabular}

Notes: Total cases 75 ; affected cases 68 (90.67\%).

Abbreviations: $\uparrow$, increase; $\downarrow$, decrease; Hb, hemoglobin; HbF, fetal hemoglobin; MCV, mean corpuscular volume; RDW, red blood cell distribution width.

Table 2 Sex wise distribution of $\mathrm{Hb}$ variants and mutations in our study

\begin{tabular}{|c|c|c|c|c|c|c|c|c|c|c|c|c|c|c|}
\hline Nos & $\begin{array}{l}\text { Age, } \\
\text { years }\end{array}$ & Sex & $\begin{array}{l}\text { HbA } \\
\text { (\%) }\end{array}$ & $\begin{array}{l}\text { MCV } \\
\text { (fL) }\end{array}$ & $\begin{array}{l}\mathrm{MCH} \\
\text { (pg) }\end{array}$ & $\begin{array}{l}\text { RDW } \\
\text { (\%) }\end{array}$ & $\begin{array}{l}\text { HbA2 } \\
\text { (\%) }\end{array}$ & $\begin{array}{l}\text { HbE } \\
\text { (\%) }\end{array}$ & $\begin{array}{l}\text { HbF } \\
\text { (\%) }\end{array}$ & $\begin{array}{l}\text { HbD } \\
\text { (\%) }\end{array}$ & Mutations & Genotype & $\begin{array}{l}\text { Inferencel } \\
\text { clinical report }\end{array}$ & Technique(s) \\
\hline I & 24 & Female & 94.30 & 75.00 & 24.00 & 14.60 & 5.00 & 0.0 & 0.70 & - & c. $92+5 G>C$ & $\mathrm{~B}^{+} / \beta$ & $\begin{array}{l}\beta \text {-thalassemia } \\
\text { minor }\end{array}$ & SS and PCR-ARMS \\
\hline 2 & 27 & Male & 94.50 & 67.00 & 18.20 & 17.00 & 4.70 & 0.0 & 0.80 & - & c.47G >A (p.Trp |6Ter) & $\mathrm{B} / \beta$ & $\begin{array}{l}\beta \text {-thalassemia } \\
\text { minor }\end{array}$ & SS \\
\hline 3 & 25 & Female & 93.95 & 69.50 & 20.80 & 17.10 & 5.40 & 0.0 & 0.65 & - & $c .92+5 G>C$ & $\mathrm{~B}^{+} / \beta$ & $\begin{array}{l}\beta \text {-thalassemia } \\
\text { minor }\end{array}$ & SS and PCR-ARMS \\
\hline 4 & 28 & Male & 93.75 & 75.50 & 18.40 & 41.30 & 5.40 & 0.0 & 0.85 & - & c. $92+5 G>C$ & $\mathrm{~B}^{+} / \beta$ & $\begin{array}{l}\beta \text {-thalassemia } \\
\text { minor }\end{array}$ & SS and PCR-ARMS \\
\hline 5 & 25 & Female & 94.61 & 69.00 & 21.00 & 17.80 & 4.80 & 0.0 & 0.59 & - & $c .92+5 G>C$ & $\mathrm{~B}^{+} / \beta$ & $\begin{array}{l}\beta \text {-thalassemia } \\
\text { minor }\end{array}$ & SS and PCR-ARMS \\
\hline 6 & 5 & Female & 65.65 & 69.90 & 18.50 & 15.10 & 33.60 & 0.0 & 0.75 & - & $c .92+5 G>C$ & $\mathrm{~B}^{+} / \beta^{+}$ & $\begin{array}{l}\beta \text {-thalassemia } \\
\text { minor }\end{array}$ & SS and PCR-ARMS \\
\hline 7 & 28 & Male & 94.35 & 68.00 & 21.00 & 18.00 & 4.70 & - & 0.95 & - & $c .92+5 G>C$ & $\mathrm{~B}^{+} / \beta$ & $\begin{array}{l}\beta \text {-thalassemia } \\
\text { minor }\end{array}$ & SS and PCR-ARMS \\
\hline 8 & 28 & Female & 93.40 & 76.40 & 23.80 & 15.60 & 5.40 & - & 1.20 & - & c. $92+5 G>C$ & $\mathrm{~B}^{+} / \beta$ & $\begin{array}{l}\beta \text {-thalassemia } \\
\text { minor }\end{array}$ & SS and PCR-ARMS \\
\hline 9 & 30 & Male & 92.50 & 75.00 & 22.40 & 14.70 & 6.60 & - & 0.90 & - & Not detected* & $\beta / \beta$ & Normal & SS and GE \\
\hline 10 & 25 & Female & 93.95 & 74.90 & 18.60 & 15.00 & 5.00 & - & 1.05 & - & c. $92+5 G>C$ & $\mathrm{~B}^{+} / \beta$ & $\begin{array}{l}\beta \text {-thalassemia } \\
\text { minor }\end{array}$ & SS and PCR-ARMS \\
\hline 11 & 30 & Male & 96.80 & 79.90 & 28.00 & 14.00 & 2.60 & - & 0.60 & - & Not detected & $\beta / \beta$ & Normal & SS and GE \\
\hline 12 & 33 & Female & 66.50 & 71.00 & 27.90 & 14.60 & 22.96 & 9.84 & 0.70 & - & c.79G>A (p.Glu27Lys) & $\beta^{\mathrm{E}} / \beta$ & $\mathrm{HbE}$ trait & SS \\
\hline 13 & 33 & Male & 92.80 & 70.10 & 20.90 & 16.90 & 6.60 & - & 0.60 & - & c.47G >A (p.Trp 16Ter) & $\beta / \beta$ & $\begin{array}{l}\beta \text {-thalassemia } \\
\text { minor }\end{array}$ & SS \\
\hline 14 & 20 & Female & 94.55 & 68.60 & 18.50 & 16.20 & 4.70 & - & 0.75 & - & $c .92+5 G>C$ & $\beta^{+} / \beta$ & $\begin{array}{l}\beta \text {-thalassemia } \\
\text { minor }\end{array}$ & SS and PCR-ARMS \\
\hline 15 & 28 & Male & 94.00 & 76.30 & 19.40 & 17.40 & 5.20 & - & 0.80 & - & c. $92+5 G>C$ & $\beta^{+} / \beta$ & $\begin{array}{l}\beta \text {-thalassemia } \\
\text { minor }\end{array}$ & SS and PCR-ARMS \\
\hline 16 & 6 & Male & 87.50 & 67.00 & 18.10 & 19.40 & 2.20 & - & 10.30 & - & c. $92+5 G>C$ & $\beta^{+} / \beta^{+}$ & $\begin{array}{l}\beta \text {-thalassemia } \\
\text { minor }\end{array}$ & SS and PCR-ARMS \\
\hline 17 & 26 & Female & 93.80 & 68.50 & 19.40 & 16.20 & 5.70 & - & 0.50 & - & 619 bp deletion & $\beta^{0} / \beta$ & $\begin{array}{l}\beta \text {-thalassemia } \\
\text { minor }\end{array}$ & GE \\
\hline 18 & 28 & Male & 95.20 & 68.30 & 20.80 & 15.50 & 4.60 & - & 0.20 & - & c. $92+5 G>C$ & $\beta^{+} / \beta$ & $\begin{array}{l}\beta \text {-thalassemia } \\
\text { minor }\end{array}$ & SS and PCR-ARMS \\
\hline 19 & I & Female & 94.90 & 70.20 & 19.20 & 16.90 & 2.70 & - & 2.40 & - & c. $92+5 G>C$ & $\beta^{+} / \beta$ & $\begin{array}{l}\beta \text {-thalassemia } \\
\text { minor }\end{array}$ & SS and PCR-ARMS \\
\hline 20 & 29 & Male & 17.90 & 67.50 & 18.40 & 16.30 & 68.90 & 12.70 & 0.50 & - & c.79G >A (p.E27K) & $\beta E / \beta$ & $\mathrm{HbE}$ trait & SS \\
\hline 21 & 24 & Female & 66.15 & 69.00 & 27.80 & 14.50 & 23.52 & 10.08 & 0.25 & - & c.79G>A (p.Glu27Lys) & $\beta^{E} / \beta$ & $\mathrm{HbE}$ trait & SS \\
\hline 22 & 31 & Male & 94.35 & 74.20 & 21.00 & 16.40 & 5.20 & - & 0.45 & - & c. $92+5 G>C$ & $\beta^{+} / \beta$ & $\begin{array}{l}\beta \text {-thalassemia } \\
\text { minor }\end{array}$ & SS and PCR-ARMS \\
\hline 23 & 27 & Female & 93.45 & 67.00 & 22.80 & 17.70 & 5.90 & - & 0.65 & - & c. $92+5 G>C$ & $\beta^{+} / \beta$ & $\begin{array}{l}\beta \text {-thalassemia } \\
\text { minor }\end{array}$ & SS and PCR-ARMS \\
\hline 24 & I & Male & 6.70 & 69.20 & 21.40 & 38.20 & 3.30 & - & 90.0 & - & $\begin{array}{l}\text { c. } 47 \mathrm{G}>\mathrm{A} \text { (p.Trp 16Ter) } \\
\text { and c. } 92+5 \mathrm{G}>\mathrm{C}\end{array}$ & $\beta^{0 /} \beta^{+}$ & $\begin{array}{l}\beta \text {-thalassemia } \\
\text { minor }\end{array}$ & SS and PCR-ARMS \\
\hline
\end{tabular}

(Continued) 
Table 2 (Continued)

\begin{tabular}{|c|c|c|c|c|c|c|c|c|c|c|c|c|c|c|}
\hline Nos & $\begin{array}{l}\text { Age, } \\
\text { years }\end{array}$ & Sex & $\begin{array}{l}\text { HbA } \\
\text { (\%) }\end{array}$ & $\begin{array}{l}\text { MCV } \\
\text { (fL) }\end{array}$ & $\begin{array}{l}\mathrm{MCH} \\
\text { (pg) }\end{array}$ & $\begin{array}{l}\text { RDW } \\
\text { (\%) }\end{array}$ & $\begin{array}{l}\text { HbA2 } \\
\text { (\%) }\end{array}$ & $\begin{array}{l}\text { HbE } \\
(\%)\end{array}$ & $\begin{array}{l}\text { HbF } \\
\text { (\%) }\end{array}$ & $\begin{array}{l}\text { HbD } \\
\text { (\%) }\end{array}$ & Mutations & Genotype & $\begin{array}{l}\text { Inferencel } \\
\text { clinical report }\end{array}$ & Technique(s) \\
\hline 25 & 30 & Male & 95.55 & 73.90 & 21.50 & 15.90 & 4.20 & - & 0.25 & - & c.47G >A (p.Trp|6Ter) & $\beta^{\circ /} \beta$ & $\begin{array}{l}\beta \text {-thalassemia } \\
\text { minor }\end{array}$ & SS \\
\hline 26 & 35 & Female & 93.70 & 69.50 & 20.80 & 17.20 & 5.70 & - & 0.60 & - & c. $92+5 G>C$ & $\beta^{+} / \beta$ & $\begin{array}{l}\beta \text {-thalassemia } \\
\text { minor }\end{array}$ & SS and PCR-ARMS \\
\hline 27 & 5 & Male & 4.00 & 66.90 & 18.50 & 15.90 & 58.80 & 25.80 & 11.40 & - & $\begin{array}{l}\text { c.79G }>\text { A (p.Glu27Lys) } \\
\text { and c. } 92+5 G>C\end{array}$ & $\beta^{0 /} \beta^{+}$ & $\begin{array}{l}\beta \text {-thalassemia } \\
\text { minor }\end{array}$ & SS and PCR-ARMS \\
\hline 28 & 30 & Female & 93.20 & 68.00 & 22.30 & 16.60 & 6.20 & - & 0.60 & - & c. $92+5 G>C$ & $\beta^{+} / \beta$ & $\begin{array}{l}\beta \text {-thalassemia } \\
\text { minor }\end{array}$ & SS and PCR-ARMS \\
\hline 29 & 32 & Female & 2.90 & 67.30 & 18.20 & 17.00 & 61.80 & 28.20 & 7.10 & - & c.79G >A (p.Glu27Lys) & $\beta^{E} \beta$ & $\mathrm{HbE}$ trait & SS \\
\hline 30 & 4 & Male & 4.00 & 69.50 & 21.60 & 37.50 & 1.10 & - & 94.90 & - & c.5IdelC & $\beta^{0 /} \beta$ & $\begin{array}{l}\beta \text {-thalassemia } \\
\text { minor }\end{array}$ & SS \\
\hline 31 & 26 & Female & 93.50 & 66.40 & 21.70 & 18.20 & 5.90 & - & 0.60 & - & c. $27 \_28$ insG & $\beta^{0 /} \beta$ & $\begin{array}{l}\beta \text {-thalassemia } \\
\text { minor }\end{array}$ & SS \\
\hline 32 & 2 & Female & 1.40 & 65.30 & 18.40 & 16.70 & 2.20 & - & 96.40 & - & c. $27 \_28$ insG & $\beta^{0} / \beta^{\circ}$ & $\begin{array}{l}\beta \text {-thalassemia } \\
\text { minor }\end{array}$ & SS \\
\hline 33 & 27 & Male & 1.55 & 75.30 & 22.80 & 16.30 & 3.80 & - & 0.25 & 94.40 & $\begin{array}{l}\text { c. } 27 \_28 \text { ins G and } \\
\text { c.364G }>\text { C (p.EI22Q) }\end{array}$ & $\beta^{\circ} / \beta^{\mathrm{D}}$ & $\begin{array}{l}\mathrm{HbD} / \beta- \\
\text { thalassemia }\end{array}$ & SS \\
\hline 34 & 27 & Female & 96.10 & 70.30 & 21.90 & 17.20 & 3.60 & - & 0.30 & - & 619 bp deletion & $\beta^{0 /} \beta$ & $\begin{array}{l}\beta \text {-thalassemia } \\
\text { minor }\end{array}$ & GE \\
\hline 35 & 30 & Male & 95.40 & 68.50 & 21.20 & 17.70 & 4.10 & - & 0.50 & - & 619 bp deletion & $\beta^{0 /} \beta$ & $\begin{array}{l}\beta \text {-thalassemia } \\
\text { minor }\end{array}$ & GE \\
\hline 36 & 27 & Female & 94.75 & 62.20 & 20.40 & 16.80 & 4.80 & - & 0.45 & - & c. $92+5 G>C$ & $\beta^{+} / \beta$ & $\begin{array}{l}\beta \text {-thalassemia } \\
\text { minor }\end{array}$ & SS and PCR-ARMS \\
\hline 37 & 30 & Male & 93.65 & 64.20 & 19.60 & 15.70 & 5.80 & - & 0.55 & - & c. $92+5 G>C$ & $\beta^{+} / \beta$ & $\begin{array}{l}\beta \text {-thalassemia } \\
\text { minor }\end{array}$ & SS and PCR-ARMS \\
\hline 38 & 23 & Female & 93.45 & 69.80 & 22.50 & 14.70 & 6.20 & - & 0.35 & - & c. $47 \mathrm{G}>\mathrm{A}$ & $\beta^{\circ} \beta$ & $\begin{array}{l}\beta \text {-thalassemia } \\
\text { minor }\end{array}$ & SS \\
\hline 39 & 26 & Male & 97.10 & 75.90 & 24.50 & 13.40 & 2.80 & - & 0.10 & - & Not detected & $\beta / \beta$ & Normal & SS and GE \\
\hline 40 & 29 & Female & 94.95 & 68.90 & 22.30 & 16.90 & 4.80 & - & 0.25 & - & $92+5 G>C$ & $\beta^{+} / \beta$ & $\begin{array}{l}\beta \text {-thalassemia } \\
\text { minor }\end{array}$ & SS and PCR-ARMS \\
\hline $4 I$ & 31 & Male & 96.10 & 69.30 & 20.50 & 15.70 & 3.90 & - & 0.00 & - & $92+5 G>C$ & $\beta^{+} / \beta$ & $\begin{array}{l}\beta \text {-thalassemia } \\
\text { minor }\end{array}$ & SS and PCR-ARMS \\
\hline 42 & 5 & Female & 94.75 & 69.70 & 22.40 & 15.80 & 5.00 & - & 0.25 & - & $92+5 G>C$ & $\beta^{+} / \beta^{+}$ & $\begin{array}{l}\beta \text {-thalassemia } \\
\text { minor }\end{array}$ & SS and PCR-ARMS \\
\hline 43 & 8 & Female & 80.00 & 68.50 & 21.60 & 16.80 & 14.00 & 6.00 & 0.00 & - & c. $79 \mathrm{G}>\mathrm{A}$ & $\beta E / \beta$ & $\mathrm{HbE}$ trait & SS \\
\hline 44 & 31 & Female & 94.27 & 64.20 & 19.50 & 15.80 & 5.28 & - & 0.45 & - & c. $92+5 G>C$ & $\beta^{+} / \beta$ & $\begin{array}{l}\beta \text {-thalassemia } \\
\text { minor }\end{array}$ & SS and PCR-ARMS \\
\hline 45 & 33 & Male & 93.00 & 69.40 & 20.40 & 17.80 & 6.60 & - & 0.40 & - & c. $92+5 G>C$ & $\beta^{+} / \beta$ & $\begin{array}{l}\beta \text {-thalassemia } \\
\text { minor }\end{array}$ & SS and PCR-ARMS \\
\hline 46 & 4 & Female & 3.70 & 67.00 & 19.20 & 15.50 & 1.60 & - & 94.70 & - & $c .92+5 G>C$ & $\beta^{+} / \beta$ & $\begin{array}{l}\beta \text {-thalassemia } \\
\text { minor }\end{array}$ & SS and PCR-ARMS \\
\hline 47 & 33 & Female & 94.40 & 69.50 & 20.70 & 17.60 & 5.40 & - & 0.20 & - & 619 bp deletion & $\beta^{\circ /} \beta$ & $\begin{array}{l}\beta \text {-thalassemia } \\
\text { minor }\end{array}$ & GE \\
\hline 48 & 35 & Male & 94.50 & 64.10 & 19.70 & 16.90 & 5.20 & - & 0.30 & - & 619 bp deletion & $\beta^{0 /} \beta$ & $\begin{array}{l}\beta \text {-thalassemia } \\
\text { minor }\end{array}$ & GE \\
\hline 49 & 30 & Female & 95.60 & 63.10 & 19.90 & 16.00 & 4.20 & - & 0.20 & - & c. $92+5 G>C$ & $\beta^{+} / \beta$ & $\begin{array}{l}\beta \text {-thalassemia } \\
\text { minor }\end{array}$ & SS and PCR-ARMS \\
\hline 50 & 32 & Male & 94.80 & 70.00 & 20.10 & 15.80 & 4.80 & - & 0.40 & - & c. $92+5 G>C$ & $\beta^{+} / \beta$ & $\begin{array}{l}\beta \text {-thalassemia } \\
\text { minor }\end{array}$ & SS and PCR-ARMS \\
\hline 51 & 26 & Female & 94.40 & 68.90 & 19.40 & 17.10 & 4.90 & - & 0.70 & - & c. $92+5 G>C$ & $\beta^{+} / \beta$ & $\begin{array}{l}\beta \text {-thalassemia } \\
\text { minor }\end{array}$ & SS and PCR-ARMS \\
\hline 52 & 26 & Male & 94.65 & 69.90 & 20.30 & 17.60 & 4.80 & - & 0.55 & - & c. $92+5 G>C$ & $\beta^{+} / \beta$ & $\begin{array}{l}\beta \text {-thalassemia } \\
\text { minor }\end{array}$ & SS and PCR-ARMS \\
\hline 53 & $1 / 2$ & Female & 94.70 & 68.10 & 18.90 & 17.90 & 2.40 & - & 2.90 & - & c. $92+5 G>C$ & $\beta^{+} / \beta$ & $\begin{array}{l}\beta \text {-thalassemia } \\
\text { minor }\end{array}$ & SS and PCR-ARMS \\
\hline 54 & 35 & Female & 91.10 & 69.50 & 20.80 & 17.00 & 8.20 & - & 0.70 & - & Not detected* & - & - & SS and GE \\
\hline 55 & 38 & Male & 91.50 & 63.10 & 19.50 & 16.00 & 7.90 & - & 0.60 & - & Not detected* & - & - & SS and GE \\
\hline
\end{tabular}


Table 2 (Continued)

\begin{tabular}{|c|c|c|c|c|c|c|c|c|c|c|c|c|c|c|}
\hline Nos & $\begin{array}{l}\text { Age, } \\
\text { years }\end{array}$ & Sex & $\begin{array}{l}\text { HbA } \\
\text { (\%) }\end{array}$ & $\begin{array}{l}\text { MCV } \\
\text { (fL) }\end{array}$ & $\begin{array}{l}\mathrm{MCH} \\
\text { (pg) }\end{array}$ & $\begin{array}{l}\text { RDW } \\
\text { (\%) }\end{array}$ & $\begin{array}{l}\text { HbA2 } \\
\text { (\%) }\end{array}$ & $\begin{array}{l}\text { HbE } \\
\text { (\%) }\end{array}$ & $\begin{array}{l}\text { HbF } \\
\text { (\%) }\end{array}$ & $\begin{array}{l}\text { HbD } \\
\text { (\%) }\end{array}$ & Mutations & Genotype & $\begin{array}{l}\text { Inferencel } \\
\text { clinical report }\end{array}$ & Technique(s) \\
\hline 56 & 4 & Female & 94.50 & 75.00 & 23.10 & 14.00 & 5.00 & - & 0.50 & - & Not detected* & - & - & SS and GE \\
\hline 57 & 5 & Female & 93.30 & 75.60 & 25.00 & 14.10 & 6.10 & - & 0.60 & - & Not detected* & - & - & SS and GE \\
\hline 58 & 22 & Female & 93.80 & 69.00 & 21.40 & 38.20 & 5.80 & - & 0.40 & - & $\begin{array}{l}\text { c. 124_I27delTTCT** } \\
\text { and c. } 92+5 \mathrm{G}>\mathrm{C}\end{array}$ & $* * / \beta^{+}$ & $\begin{array}{l}\beta \text {-thalassemia } \\
\text { minor }\end{array}$ & SS and PCR-ARMS \\
\hline 59 & 26 & Male & 94.30 & 63.20 & 19.50 & 16.10 & 5.20 & - & 0.50 & - & c. $92+5 \mathrm{G}>\mathrm{C}$ & $\beta^{+} / \beta$ & $\begin{array}{l}\beta \text {-thalassemia } \\
\text { minor }\end{array}$ & SS and PCR-ARMS \\
\hline 60 & 29 & Female & 93.55 & 67.40 & 19.10 & 17.80 & 6.10 & - & 0.35 & - & 619 bp deletion & $\beta^{0 /} \beta$ & $\begin{array}{l}\beta \text {-thalassemia } \\
\text { minor }\end{array}$ & GE \\
\hline 61 & 30 & Female & 93.95 & 69.10 & 21.80 & 17.80 & 5.50 & - & 0.55 & - & 619 bp deletion & $\beta^{0 /} \beta$ & $\begin{array}{l}\beta \text {-thalassemia } \\
\text { minor }\end{array}$ & GE \\
\hline 62 & 28 & Female & 94.00 & 69.00 & 21.40 & 38.20 & 5.40 & - & 0.60 & - & 619 bp deletion & $\beta^{0 /} \beta$ & $\begin{array}{l}\beta \text {-thalassemia } \\
\text { minor }\end{array}$ & GE \\
\hline 63 & 30 & Male & 93.60 & 69.40 & 20.70 & 17.00 & 5.90 & - & 0.50 & - & c. 27 28insG & $\beta^{0 /} \beta$ & $\begin{array}{l}\beta \text {-thalassemia } \\
\text { minor }\end{array}$ & SS \\
\hline 64 & 27 & Female & 94.40 & 69.00 & 21.80 & 17.40 & 5.20 & - & 0.40 & - & c. $92+5 \mathrm{G}>\mathrm{C}$ & $\beta^{+} / \beta$ & $\begin{array}{l}\beta \text {-thalassemia } \\
\text { minor }\end{array}$ & SS and PCR-ARMS \\
\hline 65 & 30 & Male & 94.30 & 67.10 & 18.20 & 19.10 & 5.00 & - & 0.70 & - & c. $27 \_28$ insG & $\beta^{0 /} \beta$ & $\begin{array}{l}\beta \text {-thalassemia } \\
\text { minor }\end{array}$ & SS \\
\hline 66 & 24 & Male & 93.20 & 69.00 & 21.70 & 17.80 & 6.10 & - & 0.70 & - & $619 \mathrm{bp}$ deletion & $\beta^{0 /} \beta$ & $\begin{array}{l}\beta \text {-thalassemia } \\
\text { minor }\end{array}$ & GE \\
\hline 67 & 26 & Male & 91.75 & 62.40 & 19.90 & 18.20 & 7.90 & - & 0.35 & - & c. $92+5 G>C$ & $\beta^{+} / \beta$ & $\begin{array}{l}\beta \text {-thalassemia } \\
\text { minor }\end{array}$ & SS and PCR-ARMS \\
\hline 68 & 26 & Female & 94.35 & 68.90 & 20.90 & 17.60 & 4.90 & - & 0.75 & - & c. $92+5 G>C$ & $\beta^{+} / \beta$ & $\begin{array}{l}\beta \text {-thalassemia } \\
\text { minor }\end{array}$ & SS and PCR-ARMS \\
\hline 69 & 23 & Male & 93.80 & 73.90 & 20.10 & 14.60 & 5.50 & - & 0.70 & - & $c .92+5 G>C$ & $\beta^{+} / \beta$ & $\begin{array}{l}\beta \text {-thalassemia } \\
\text { minor }\end{array}$ & SS and PCR-ARMS \\
\hline 70 & 27 & Female & 94.40 & 74.30 & 19.30 & 15.90 & 5.00 & - & 0.60 & - & c. $92+5 \mathrm{G}>\mathrm{C}$ & $\beta^{+} / \beta$ & $\begin{array}{l}\beta \text {-thalassemia } \\
\text { minor }\end{array}$ & SS and PCR-ARMS \\
\hline 71 & 24 & Male & 0.0 & 72.90 & 21.00 & 14.50 & 3.40 & - & 96.60 & - & $\begin{array}{l}\text { c.5I_5IdelC and } \\
\text { c. } 124 \_ \text {_27delTTCT** }\end{array}$ & $\beta^{0 / * *}$ & $\begin{array}{l}\beta \text {-thalassemia } \\
\text { minor }\end{array}$ & SS \\
\hline 72 & 26 & Male & 94.20 & 68.40 & 21.00 & 14.00 & 5.20 & - & 0.60 & - & c. $92+5 G>C$ & $\beta^{+} / \beta$ & $\begin{array}{l}\beta \text {-thalassemia } \\
\text { minor }\end{array}$ & SS and PCR-ARMS \\
\hline 73 & 27 & Female & 69.80 & 74.20 & 20.30 & 14.40 & 20.73 & 8.97 & 0.50 & - & c.79G >A (p.E27K) & $\beta E / \beta$ & HbE trait & SS \\
\hline 74 & 28 & Male & 68.40 & 70.20 & 21.80 & 16.90 & 21.70 & 9.30 & 0.60 & - & c.79G >A (p.E27K) & $\beta^{\mathrm{E} / \beta}$ & HbE trait & SS \\
\hline 75 & 27 & Male & 95.40 & 64.20 & 20.10 & 16.40 & 3.90 & - & 0.70 & - & $c .92+5 G>C$ & $\beta^{+} / \beta$ & $\begin{array}{l}\beta \text {-thalassemia } \\
\text { minor }\end{array}$ & SS and PCR-ARMS \\
\hline
\end{tabular}

Notes: Total cases: 75 ; age range (I/2 to 38 years). Case nos: $24,27,33$, 58, and $7 \mathrm{I}$ had double mutations (compound heterozygous). Mean $\mathrm{HbA}=8 \mathrm{I} .37$ ( $96.8 \%-97.8 \%$ ), mean $\mathrm{MCV}=69.53(83-100 \mathrm{fL})$, mean $\mathrm{MCH}=20.92(27-32 \mathrm{pg})$, mean RDW = $17.85(1 \mathrm{I} .5 \%-14.5 \%)$, mean $\mathrm{HbA2}=8.66(2 \%-3.5 \%)$, mean HbE = 2.73\% (absent), HbD = $1.25 \%$ (absent), and $\mathrm{HbF}=7.21$ ( $0.0 \%-1.0 \%)$. Figures in parentheses indicate normal range/values.

*Large deletion and duplication are not identified in our study. **Genotype novel.

Abbreviations: GE, gel electrophoresis; Hb, hemoglobin; MCV, mean corpuscular volume; PCR-ARMS, polymerase chain reaction-amplification refractory mutation system; RDW, red blood cell distribution width; SS, Sanger sequencing.

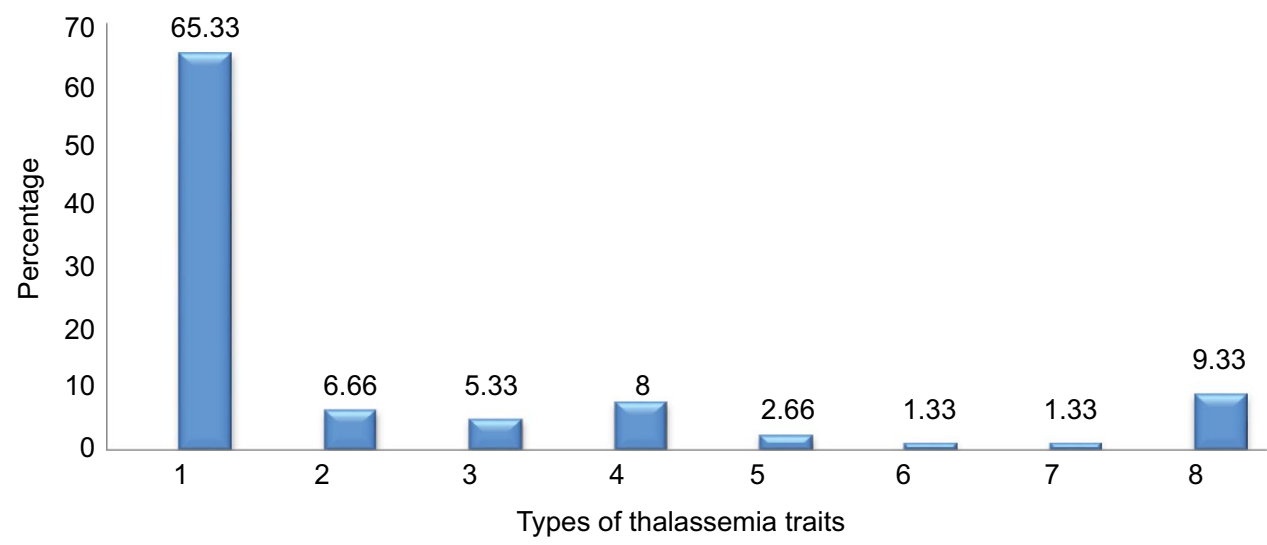

Figure I Percentage (\%) distribution of $\beta$-thalassemia traits. 


\section{Mutation analysis}

We have analyzed conventional mutations of eight in Indian population using PCR-ARMS and end point PCR allayed with using Codon code Aligner V6.0.2 and mutation survey 5.0 software for exact specific mutation nomenclature from 68 affected cases. The data showed that c. $92+5 \mathrm{G}>\mathrm{C}$ was higher $(41,60.29 \%)$, followed by nine cases of deletion 619 bp (13.23\%), eight cases of c.79G>A (p.E27K) (11.76\%), and five cases each of c.27_28insG (7.35\%) and c. $47 \mathrm{G}>\mathrm{A}$ (p.Trp16Ter) (7.35\%) with two cases each of c.124_127delTTCT (2.94\%) and c.51delC (2.94\%) and one case each of c.364G>C (p.E122Q) and c.47G $>A$ (p.Trp16Ter) (1.47\%), respectively, with no sex difference as female and male ratio was (1:1.13) (Table 3). Deletion 619 bp was only detected by gel electrophoresis, and c. $92+5 \mathrm{G}>\mathrm{C}$ was identified using PCR-ARMS and also gene sequencing as that of others (Figure $2 \mathrm{~A}-\mathrm{G}$ ). In Rajasthan and Gujarat, where more are accumulated (40 and 17) respectively, in both cases, the most frequent mutation is c. $92+\mathrm{G}>\mathrm{C}$ (26 and 10) followed by 619 bp ( 3 and 4 ) and 619 bp deletions (Table 3 and Figure 3).

\section{Discussion}

$\beta$-Thalassemia is one of the heterozygous inheritable disorders in India. It causes reduced or absence of $\beta$-chain synthesis of $\mathrm{Hb}$. Its variants in addition to carrier identification and prenatal analysis are necessary for its management and to avoid marriages between carrier of mutated genes including consanguineous types. ${ }^{4,10-12}$ Hence, from 75 referral cases of Western and Eastern India, the blood was collected to identify various traits and mutations accurately using electrophoretic and molecular diagnostic techniques in our laboratory including coinheritance with $\beta$-thalassemia. Of the total referral patients, 68 cases were affected having $90.67 \%$ in this study. Of these, $65.33 \%$ of $\beta$-thalassemia traits (49) were detected followed by $\mathrm{HbE}$ trait and $\beta$-thalassemia major with $\mathrm{HbE}$ homozygous and their $\mathrm{HbE} / \beta$-thalassemia and $\mathrm{HbD} / \beta$-thalassemia coinherited cases depending on altered $\mathrm{Hb}, \mathrm{MCH}$, red blood cell distribution width, and MCV values. It indicated that $\beta$-thalassemia cases (carriers) are maximum followed by others and support the data of previous workers in India. ${ }^{13-16}$ Similarly, $\mathrm{Hb}$ patterns were measured and presented in the study of Mondal and Mandal, ${ }^{8}$ who obtained few cases of $\mathrm{HbE}, \mathrm{HbD}$ traits, and $\beta$-thalassemia major comparatively. This could be due to changing lifestyles, environmental and genetic factors, and coinheritance of $\mathrm{HbE}, \mathrm{HbD}$, and/ or $\alpha$-thalassemia with $\beta$-thalassemia carriers. ${ }^{17,18}$ Further, these factors may also be the cause of $\beta$-thalassemia major with heterozygosity/homozygosity who are less in number requiring blood transfusion. Similarly, coinheritance of $\mathrm{HbE} /$ $\beta$-thalassemia and $\mathrm{HbD} / \beta$-thalassemia and $\mathrm{HbE}$ homozygous cases were also reduced in number in our report. HbE trait had six cases having less severity of clinical condition. However, Olivieri et a $\mathrm{l}^{17}$ mentioned that these conditions may vary from severe to mild depending upon genetic and environmental factors, and such patients are also less frequent to support our data. We detected one each of $\mathrm{HbD} / \beta$-thalassemia and $\mathrm{HbE} / \beta$-thalassemia cases in addition to $\mathrm{HbE}$ patients with variable phenotypic indices expressing mild heterozygous state. ${ }^{19-22}$ These patients may require transfusion in severe condition only, due to coinheritance of the disease..$^{23}$

Further, we extended our investigation on molecular analysis of mutations of $\beta$-thalassemia and systematically using latest molecular biology tools such as PCR-ARMS, end point

Table 3 Percentage of mutation types in our thalassemic cases (68) of different regions

\begin{tabular}{|c|c|c|c|c|c|c|}
\hline Nos & Mutation types & Mutation nos (73)* & Male (34) & Female (39) & Mutation percentage & Region (state) wise mutation \\
\hline \multirow[t]{2}{*}{$\mathrm{I}$} & c. $92+5 G>C$ & 41 & 18 & 23 & 60.29 & Rajasthan 26, Gujarat 10, West \\
\hline & & & & & & Bengal 3, Maharashtra 2 \\
\hline \multirow[t]{2}{*}{2} & 619 bp deletion & 9 & 3 & 6 & 13.23 & Gujarat 4, Rajasthan 3, Maharashtra \\
\hline & & & & & & 2 \\
\hline \multirow[t]{2}{*}{3} & c.79G >A (p.E27K) & 8 & 4 & 4 & 11.76 & Assam 3, Rajasthan 2, Maharashtra \\
\hline & & & & & & I, West Bengal 2 \\
\hline \multirow[t]{2}{*}{4} & c.47G>A (p.Trp I6Ter) & 5 & 4 & I & 7.35 & Gujarat 2, Rajasthan 2, Maharashtra \\
\hline & & & & & & I \\
\hline 5 & $c .364 G>C(p . E \mid 22 Q)$ & I & 1 & 0 & 1.47 & Rajasthan I \\
\hline \multirow[t]{2}{*}{6} & c.27_28insG & 5 & 2 & 3 & 7.35 & Rajasthan 2, Gujarat I, Maharashtra \\
\hline & & & & & & I, West Bengal I \\
\hline 7 & c.5IdelC & 2 & 2 & 0 & $\mathrm{I} .47$ & Rajasthan 2 \\
\hline 8 & c.124_I27delTTCT & 2 & 0 & 2 & $\mathrm{I} .47$ & Rajasthan 2 \\
\hline
\end{tabular}

Notes: State/region wise mutations: Rajasthan: 40, Gujarat: 17, Maharashtra: 7 (Western India: $40+17+07=64$ ), West Bengal: 6, Assam: 3 (Eastern India: $06+03=09$ ). *Five with compound heterozygous (double mutations); $\mathrm{M}: \mathrm{F}=1: 1.13(32 / 36=68)$.

Abbreviations: $F$, female; $M$, male. 


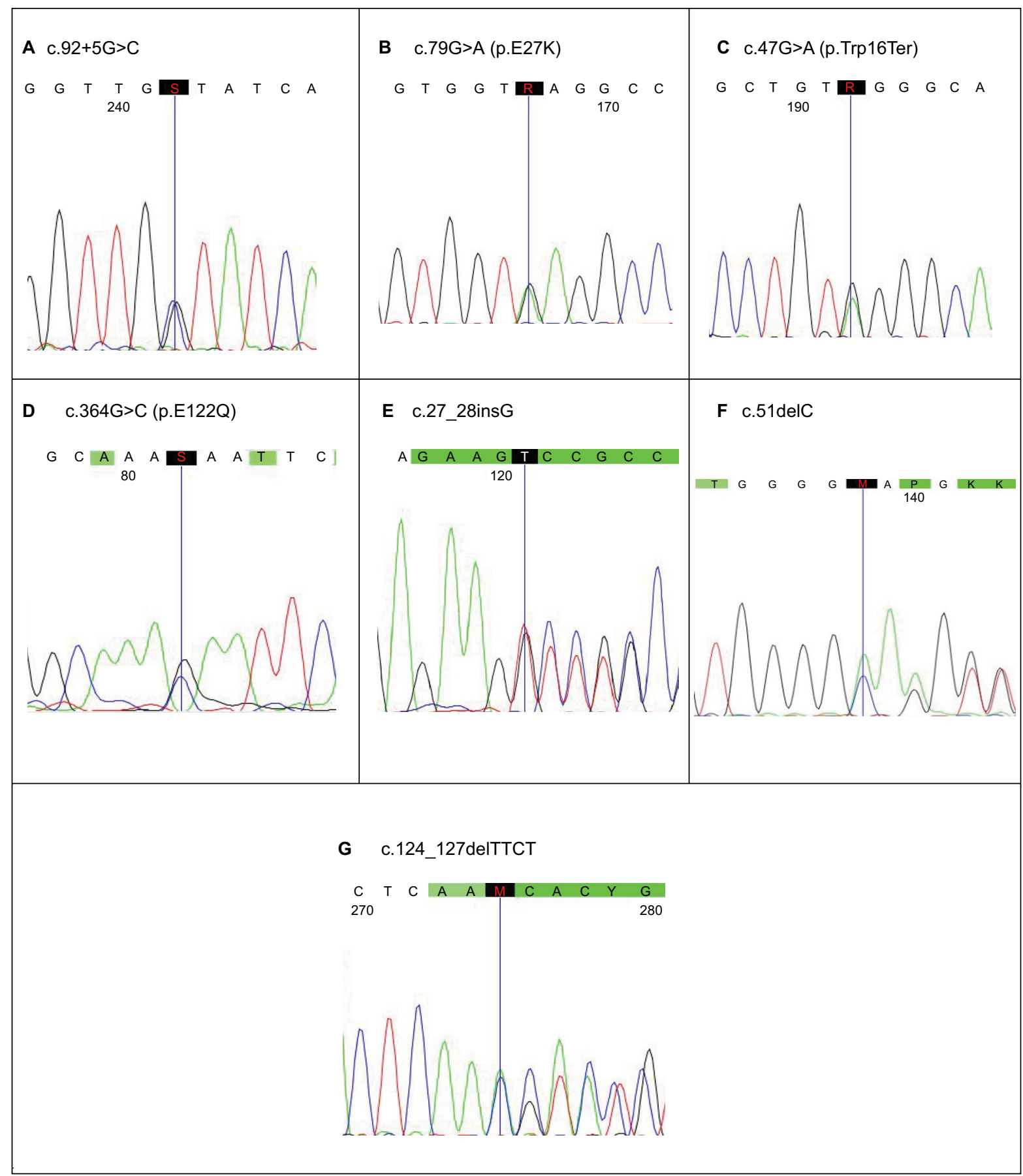

Figure 2 Seven common mutations identified by direct DNA sequencing.

Notes: (A) c.92+5G>C, (B) c.79G>A (p.E27K), (C) c.47G>A (p.Trpl6 Ter), (D) c.364G>C (p.EI22Q), (E) c.27_28insG, (F) c.5IdelC, and (G) c.124_127delTTCT. Nucleotide colors: $A=$ green, $T=$ red, $G=$ black, and $C=$ blue.

Abbreviations: S, G/C; R, G/A; K, G/T; M, C/A; Y, C/T.

PCR, and Sanger Gene Sequencing. Data revealed 92+5 G>C (IVS-1-5) is the maximum in cases $(60.29 \%)$, of Rajasthan and Gujarat followed by deletion $619 \mathrm{bp}$ and is conformed with others documented earlier in Gujarat, Maharashtra, and Rajasthan. ${ }^{2,9,12,15,24-26}$ But Hassan et al, ${ }^{27}$ from Thailand, found cd26 (A-G) $\mathrm{HbE}$ and cd41/42 (-TTCT) were higher in their studies. Thong et $\mathrm{al}^{28}$ presented cd41/42 (-TTCT) and IVS-2 654 (C-T) were maximum in Chinese population.
Similarly, second highest mutation was 619 bp in $(9,13.23 \%)$ this study similar to that of other studies in Western India conducted by Sheth et al, ${ }^{10}$ Grow et al, ${ }^{13}$ Colah et al, ${ }^{29}$ and Nigam et al. ${ }^{16}$ The third largest mutation in our study was c. 79G $>$ A (p.E27K) followed by c.47G $>$ A (p.Trp16Ter) and c.27_28insG different from other investigators, ${ }^{2}$ followed by other mutations, ie, c.51delC, c.124_127delTTCT(novel), and c.364G $>C$ (p.E122Q). The incidence of these mutations 


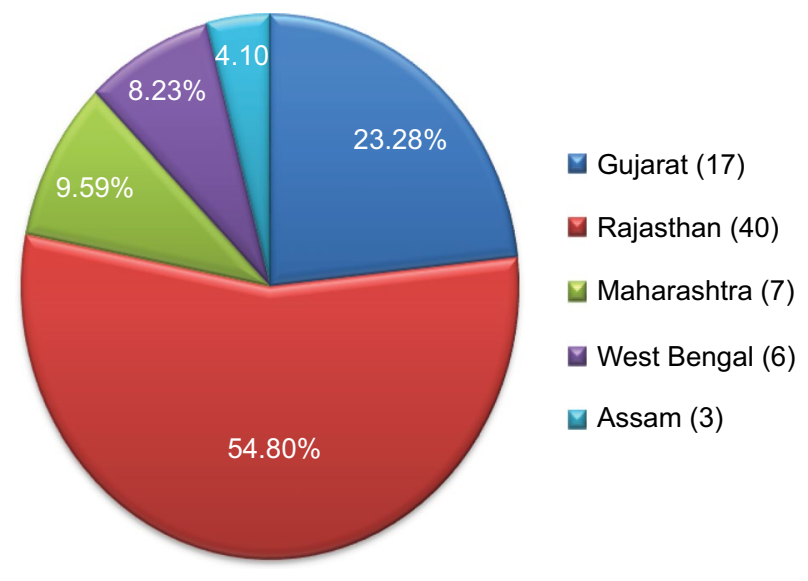

Figure 3 Region wise percentage distribution of mutations in $\beta$-thalassemia and numbers in parentheses indicate cases.

does not seem to be related to sex, as our sex ratio was 1:1.13 (M:F). The variation in occurrence of these mutations is related to regional, ethical, migration, interracial marriages, study plan, and other factors as mentioned by others. ${ }^{12,29,30}$

\section{Conclusion}

Our study showed that 68 cases were affected by $\beta$-thalassemia in our referral cases (75), from East-Western Indian region. $\beta$-Thalassemia carriers were 49 (65.33\%) followed by $\mathrm{HbE}$ trait and $\beta$-thalassemia major with heterozygous and homozygous condition using hematological profiles. Detection of molecular analysis of mutations using PCR-ARMS, end point PCR, and gene sequencing methods revealed c. $92+5 \mathrm{G}>\mathrm{C}$ mutation exhibited higher incidence $(26+10+2)$ followed by deletion 619 bp $(3+4+2)$ in Rajasthan, Gujarat, and Maharashtra (Western India) as compared to West Bengal and Assam (3:0; 0:0) of Eastern India, respectively. This requires further elucidation. The variation in incidence of these mutations is dependent on ethnic diversity, migration, genetic factors, and other lifestyles. We, hence, recommend the mass screening, Prenatal Diagnostic Techniques, genetic counseling, transfusion programs and clinical management made available to these populations before adopting assisted reproductive and preimplantation technologies in India.

\section{Acknowledgments}

The authors are thankful to all the staff including Clinicians of Supratech Micropath Laboratory, Ahmedabad, for their continuous assistance in this work. Parth S Shah, Nidhi D Shah, and Sandip C Shah are son, daughter-in-law, and father, respectively, who run this research institute.

\section{Author contributions}

Parth S Shah and Nidhi D Shah have contributed to writing results and discussion in the manuscript preparation when they visit India. Hari P Ray, Nikunj B Khatri, Ketan K Vaghasia, and Rutvik $\mathrm{J}$ Raval are involved in collection of blood from the patients after duly filled consent forms, blood analysis, DNA extraction, DNA sequencing, PCR-ARMS, end point PCR, and data analysis of 75 patients. Dr Sandip C Shah and Dr Mandava V Rao have contributed to preparation of reports after finalization of the results and preparation of the manuscript finally for submission to the journal. All authors contributed toward data analysis, drafting and critically revising the paper and agree to be accountable for all aspects of the work.

\section{Disclosure}

The authors report no conflicts of interest in this work.

\section{References}

1. Weatherall DJ, Clegg JB. Inherited haemoglobin disorders: an increasing global health problem. Bull World Health Organ. 2001;79(8):704-712.

2. Ansari MI, Patel NG. Characterization of $\beta$-thalassemia mutations from north Maharashtra region. J Pharm Biol Sci. 2015;10(3):13-16.

3. Cooley TB, Lee P. A series of cases of splenomegaly in children with anemia and peculiar bone changes. Trans Am Pediatr Soc. 1925;37: 29-30.

4. Rao MV, Shah SR, Patel AP. $\beta$-thalassemia. In: Gupta PD, Srivastava LM, editors. Essentials of Inborn Metabolic and Genetic Disorders. Chennai: Pug Publication Pvt Ltd; 2015:169-179.

5. Boonyawat B, Monsereenusorn C, Traivaree C. Molecular analysis of beta-globin gene mutations among thai beta-thalassemia children: results from a single center study. Appl Clin Genet. 2014;7: 253-258.

6. Taghavi BM, Karimipoor M, Amirian A, et al. Co-inheritance of hemoglobin D and B-thalassemia trait in three Iranian families: clinical relevance. Arch Iran Med. 2011;14(1):61-63.

7. Abolghasemi H, Amid A, Zeinali S, et al. Thalassemia in Iran: epidemiology, prevention and management. J Pediatr Hematol Oncol. 2007; 29(4):233-238

8. Mondal SK, Mandal S. Prevalence of thalassemia and hemoglobinopathy in eastern India: a 10-year high-performance liquid chromatography study of 119,336 cases. Asian J Transfus Sci. 2016;10(1):105-110.

9. Patel AP, Naik MR, Shah NM, Sharma NP, Parmar PH. Prevalence of common hemoglobinopathies in Gujarat: an analysis of a large population screening program. Natl J Comm Med. 2012;3(1):112-116.

10. Sheth JJ, Sheth FJ, Pandya P, et al. Beta thalassaemia mutations in western India. Ind J Pediatr. 2008;6:567-570.

11. Mishra AK, Tiwari A. Screening and molecular characterization of $\beta$-thalassaemia mutations in parents and siblings of $\beta$-thalassaemia major patients. Ind J Basic Appl Medl Res. 2014;5(2):481-486.

12. Cao A, Galanello R. Beta-thalassemia. Genet Med. 2010;12(2):61-76.

13. Grow K, Vashist M, Abrol P, Sharma S, Yadav R. Beta thalassemia in India: current status and the challenges ahead. Int J Pharm Pharm Sci. 2014;6(4):28-33.

14. Balgir RS. The burden of haemoglobinopathies in India and the challenges ahead. Curr Sci. 2000;79(11):1536-1547.

15. Satpute SB, Bankar MP, Momin AA. The prevalence of $\beta$-thalassemia mutations in south western Maharashtra. Ind J Clin Biochem. 2012;27(4):389-393.

16. Nigam N, Munshi M, Patel M, Soni A. Distribution of beta thalassemia mutation and its correlation with alpha thalassemia in Gujarati families. Int J Hum Genet. 2003;3(4):221-224.

17. Olivieri NF, Pakbaz Z, Vichinsky E. Hb E/beta-thalassaemia: a common \& clinically diverse disorder Indian. J Med Res. 2011;134(4):522-531. 
18. Colah R, Gorashekar A, Phanasgaonkar S, et al. Epidemiology of beta thalassemia in western India: mapping the frequencies \& mutations in subversion of Maharashtra and Gujarat. Br J Haematol. 2010;149(5): 739-747.

19. Burtis CA, Ashwood ER, Bruns DE. Tietz Textbook of Clinical Chemistry and Molecular Diagnostics. 5th ed. India: Elsevier; 2012.

20. Williamson MA, Snyder LM. Wallach's Interpretation of Diagnostic Tests. 9th ed. New York, NY: Lippincott Pub; 2011.

21. Theodoridou S, Alemayechou M, Perperidou P, Sinopoulou C, Karafoulidou T, Kiriakopoulou G. Compound heterozygosity for $\mathrm{Hb}$ D-Punjab/b-thalassemia and blood donation: case report. Turk J Hematol. 2009;26(2):100-101.

22. Rahimi Z, Akramipour R, Korani S, Nagel RL. Hb D-Punjab [beta 121 $(\mathrm{GH} 4) \mathrm{Glu} \rightarrow \mathrm{Gln}$ ]/beta0-thalassemia [IVSII.1 $(\mathrm{G} \rightarrow \mathrm{A})]$ in two cases from an Iranian family: first report. Am J Hematol. 2006;81(4):302-303.

23. Menzel S, Garner C, Gut I, et al. A QTL influencing F cell production maps to a gene encoding a zinc-finger protein on chromosome $2 \mathrm{p} 15$. Nat Genet. 2007;39(10):1197-1199.

24. Panja A, Ghosh TK, Basu A. Genetics of thalassemia in Indian population. J Community Nutr Health. 2012;1(1):39-46.
25. Bhukhanvala DS, Italia K, Sawant P, Colah R, Ghosh K, Gupte SC Molecular characterization of $\beta$-thalassemia in four communities in South Gujarat-codon $30(\mathrm{G} \rightarrow \mathrm{A})$ a predominant mutation in the Kachhiya Patel community. Ann Hematol. 2013;92(11):1473-1476.

26. Colah RB, Gorakshakar AC, Nadkarni AH. Invasive \& noninvasive approaches for prenatal diagnosis of haemoglobinopathies: experiences from India. Ind J Med Res. 2011;134(4):552-560.

27. Hassan S, Ahmad R, Zakaria Z, Zulkafli Z, Abdullah WZ. Detection of $\beta$-globin gene mutations among $\beta$-thalassaemia carriers and patients in Malaysia: application of multiplex amplification refractory mutation system-polymerase chain reaction. Malays J Med Sci. 2013; 20(1):13-20.

28. Thong MK, Tan JA, Tan KL, Yap SF. Characterisation of beta-globin gene mutation in Malaysian children: a strategy for the control of betathalassemia in a develop country. J Trop Pediatr. 2005;51(6):328-333.

29. Colah R, Gorakshakar A, Nadkarni A, et al. Regional heterogeneity of beta-thalassemia mutations in the multi ethnic Indian population. Blood Cells Mol Dis. 2009;42(3):241-246.

30. Nadkarni AH, Nair SB, Italia KY, et al. Molecular diversity of hemoglobin H disease in India. Am J Clin Pathol. 2010;133(3):491-494.
The Application of Clinical Genetics

\section{Publish your work in this journal}

The Application of Clinical Genetics is an international, peer-reviewed open access journal that welcomes laboratory and clinical findings in the field of human genetics. Specific topics include: Population genetics; Functional genetics; Natural history of genetic disease; Management of genetic disease; Mechanisms of genetic disease; Counselling and ethical

\section{Dovepress}

issues; Animal models; Pharmacogenetics; Prenatal diagnosis; Dysmorphology. The manuscript management system is completely online and includes a very quick and fair peer-review system, which is all easy to use. Visit http://www.dovepress.com/testimonials.php to read real quotes from published authors. 